\title{
Preservation of Genetic Diversity of the Asian Native Goats
}

\author{
Takeshi Honda $^{1, ~}$, Mayu Shibano ${ }^{1}$, Hirokazu Matsumoto ${ }^{2}$, Shinji Sasazaki ${ }^{3}$, Kenji Oyama ${ }^{1}$, \\ Hideyuki Mannen ${ }^{3}$
}

${ }^{1}$ Food Resources Education and Research Center, Graduate School of Agricultural Science, Kobe University, Hyogo, Japan

${ }^{2}$ Laboratory of Animal Genetics, Department of Animal Science, School of Agriculture, Tokai University, Kumamoto, Japan

${ }^{3}$ Laboratory of Animal Breeding and Genetics, Graduate School of Agricultural Science, Kobe University, Kobe, Japan

Email address:

takhonda@diamond.kobe-u.ac.jp (T. Honda)

${ }^{*}$ Corresponding author

\section{To cite this article:}

Takeshi Honda, Mayu Shibano, Hirokazu Matsumoto, Shinji Sasazaki, Kenji Oyama, Hideyuki Mannen. Preservation of Genetic Diversity of the Asian Native Goats. Animal and Veterinary Sciences. Vol. 5, No. 5, 2017, pp. 69-72. doi: 10.11648/j.avs.20170505.12

Received: August 2, 2017; Accepted: August 23, 2017; Published: September 22, 2017

\begin{abstract}
Using single nucleotide polymorphisms (SNPs), the relative importance of 10 subpopulations of Asian native goats in preserving genetic diversity was investigated. Analysis of prioritizing by removal of subpopulations identified the subpopulations of Mongolia (MGL), Myanmar (MYA), Cambodian plains (CAM_P), India (IND), and Philippine (PHI) as genetically important subpopulations because their removal resulted in a $6.38 \%$ reduction of expected heterozygosity. The removal of the remaining five subpopulations resulted in a $1.45 \%$ increase. Likewise, analysis using the core set method identified five subpopulations (MGL, MYA, CAM_P, IND, and PHI) as genetically important subpopulations. Among these five subpopulations, the IND was most important because of low molecular coancestry within itself and between the other subpopulations. The subpopulations of Cambodian mountainous (CAM_M) and Vietnam (VIE) were also considered to be important in this analysis. Based on these two investigations, we concluded that MGL, MYA, CAM_P, IND, and PHI are essential, and that CAM_M and VIE are worth preserving.
\end{abstract}

Keywords: Capra Hircus, Single Nucleotide Polymorphism, Molecular Coancestry, Expected Heterozygosity, Core Set Method

\section{Introduction}

Goats are raised all over the world, and more than $65 \%$ of these goats are in developing countries. The production of meat, milk, and hide is very important and supports the lives of people in those countries. Although Asia has rich native goat resources, these animals have been crossed with imported profitable breeds, and face the risk of extinction [1]. Therefore, proposing a specific preservation scheme for native goat genetic resources in Asia is an urgent task.

Recently, the origin, phylogeny, and genetic diversity of Asian native goats were studied using various molecular genetic techniques ([2], [3], [4]). However, genetically important subpopulations for preserving the genetic variability of Asian native goats have not yet been identified. Prioritizing subpopulations is an essential task because both human and economic resources for the preservation schemes are usually limited, and should be conducted before the establishment of the preservation scheme. In this study, we identified genetically important goat subpopulations among 10 subpopulations from Asia (Mongolia (MGL), Laos (LAO), Myanmar (MYA), Cambodian mountainous (CAM_M), Cambodian plains (CAM P), Vietnam (VIE), Bhutan (BHU), Bangladesh (BAN), India (IND), and Philippine (PHI)), using 65 single nucleotide polymorphism (SNP) markers developed by Lin et al. [3].

\section{Materials and Methods}

\subsection{Animals}

Thirty animals were sampled from each subpopulation except for IND, from which 20 animals were sampled. Genomic DNA was extracted from blood according to the standard phenol and chloroform method. 


\subsection{SNP Detection}

For detailed information about the process of detection of SNP markers, see Lin et al. [3].

\subsection{Population Genetic Analysis}

Caballero \& Toro [5] proposed a method for genetically prioritizing the subpopulations, in which the change (decrease or increase) of expected heterozygosity [6] is examined when one or more subpopulations are assumed to be removed from the whole population. Assuming an equal subpopulation size and letting $f_{i j}$ be the molecular coancestry between subpopulations $i$ and $j$, average molecular coancestry of the whole population $(\bar{f})$ can be decomposed into

$$
\bar{f}=\tilde{f}-\overline{\mathrm{D}}
$$

where $\tilde{f}$ is the average of within-subpopulation coancestry $\left(f_{i i}\right)$ and $\overline{\mathrm{D}}$ is the average of Nei's minimum distance $\left([7]: \mathrm{D}_{i j}=\right.$ $\left.\left(f_{i i}+f_{j j}\right) / 2-f_{i j}\right)$ among all the subpopulations. Equation (1) indicates that the average molecular coancestry of the whole population $(\bar{f})$ depends on the within-subpopulation component $(\tilde{f})$ and averaged distance among the subpopulations $(\overline{\mathrm{D}})$. Thus, the total genetic diversity of the whole population $\left(G D_{\mathrm{T}}\right)$ expressed as expected heterozygosity is also decomposed as

$$
G D_{T}=1-\bar{f}=(1-\tilde{f})+\overline{\mathrm{D}}=G D_{W S}+G D_{B S},
$$

implying that the total genetic diversity of the whole population is partially ascribed to within-diversity components of each subpopulation $\left(G D_{\mathrm{WS}}\right)$ and to between-diversity components among all the pairs of subpopulations $\left(G D_{\mathrm{BS}}\right)$.

In a second investigation for prioritizing the 10 subpopulations, optimal genetic contributions of subpopulations for maximizing the genetic diversity of a hypothetical gamete pool were calculated. This calculation was conducted using the core set method of Eding et al. [8], which was originally proposed for ranking animal breeds regarding conservation of genetic diversity within a livestock species. The similar technique is used in many situations nowadays (e.g. [9]). Suppose $g_{i}$ gametes are sampled from subpopulation $i$ to obtain a hypothetical gamete pool with $g_{T}$ total gametes, so that the genetic contribution of subpopulation $i$ is $c_{i}=g_{i} / g_{T}$ and

$$
\sum_{i=1}^{n} c_{i}=1
$$

Letting $f_{i j}$ be the molecular coancestry between subpopulations $i$ and $j$, and $\mathrm{F}=\left[f_{i j}\right]$ be the molecular coancestry matrix of all the subpopulations, the expected molecular coancestry of the hypothetical gamete pool is

$$
\overline{f_{c}}=\mathrm{c}^{\prime} \mathrm{Fc},
$$

where $\mathrm{c}$ is a column vector of $c_{i}$. Thus, maximization of genetic diversity of the hypothetical gamete pool $\left(1-\bar{f}_{c}\right)$ is equivalent to the problem of finding optimal $\mathrm{c}\left(\mathrm{c}_{\mathrm{opt}}\right)$ such that $\bar{f}_{c}$ is minimized under the restriction of Eq. (2). This problem can be solved by applying the Lagrange multiplier method, and the optimal contribution of each subpopulation is obtained as

$$
\mathrm{c}_{\mathrm{opt}}=\frac{\mathrm{F}^{-1} 1}{1 \mathrm{~F}^{-1} 1}
$$

where 1 is a unit vector (for detailed derivation, see Eding et al. [8]).

All the computations were conducted using our original Fortran programs.

\section{Results}

Genetic diversity and its proportional changes according to the removal of subpopulations are provided in Table 1 . The genetic diversity of the whole population $\left(G D_{\mathrm{T}}\right)$ was 0.388 , and it was decomposed into 0.331 for the within-diversity component $\left(G D_{\mathrm{WS}}\right)$ and 0.057 for the between-diversity component $\left(G D_{\mathrm{BS}}\right)$. Removal of MGL, MYA, CAM_P, IND, and $\mathrm{PHI}$ was accompanied by the reduction of $G D_{\mathrm{T}}$, indicating that these five subpopulations are genetically more important

\begin{tabular}{|c|c|c|c|c|c|c|}
\hline \multirow[b]{2}{*}{ Removed subpopulation } & \multirow[b]{2}{*}{$G D_{\mathrm{wS}}$} & \multicolumn{5}{|c|}{ Changes (\%) } \\
\hline & & $G D_{\mathrm{BS}}$ & $G D_{\mathrm{T}}$ & $G D_{\mathrm{ws}}$ & $G D_{\mathrm{BS}}$ & $G D_{\mathrm{T}}$ \\
\hline None $^{\mathrm{a}}$ & 0.331 & 0.057 & 0.388 & - & - & - \\
\hline MGL & 0.329 & 0.056 & 0.385 & -0.55 & -0.29 & -0.84 \\
\hline MYA & 0.327 & 0.061 & 0.388 & -1.17 & 1.06 & -0.11 \\
\hline CAM_M & 0.341 & 0.049 & 0.389 & 2.43 & -2.11 & 0.32 \\
\hline CAM_P & 0.329 & 0.058 & 0.387 & -0.54 & 0.32 & -0.22 \\
\hline BHU & 0.328 & 0.060 & 0.388 & -0.79 & 0.79 & 0.00 \\
\hline BAN & 0.331 & 0.059 & 0.390 & -0.11 & 0.54 & 0.44 \\
\hline IND & 0.328 & 0.054 & 0.382 & -0.84 & -0.76 & -1.59 \\
\hline PHI & 0.335 & 0.052 & 0.387 & 1.06 & -1.40 & -0.34 \\
\hline MGL+MYA+CAM_P+IND+PHI & 0.317 & 0.047 & 0.363 & -3.67 & -2.72 & -6.38 \\
\hline $\mathrm{LAO}+\mathrm{CAM} \mathrm{M}+\mathrm{VIE}+\mathrm{BHU}+\mathrm{BAN}$ & 0.345 & 0.049 & 0.394 & 3.67 & -2.21 & 1.45 \\
\hline
\end{tabular}
than the remaining five subpopulations for preserving genetic diversity.

Table 1. Total genetic diversity of the whole population (GDT), within-subpopulation component (GDWS), and between-subpopulation component (GDBS), according to the removal of subpopulations and their proportional changes in percentages. 
The reduction of $G D_{\mathrm{T}}$ caused by the simultaneous removal of the previous five subpopulations (MGL, MYA, CAM_P, IND, and PHI) (6.38\%) was substantially larger than the sum of individual effects $(3.10 \%)$. In addition, removal of the remaining five subpopulations (LAO, CAM_M, VIE, BHU, and BAN) increased $G D_{\mathrm{T}}(1.45 \%)$, indicating that all five subpopulations (MGL, MYA, CAM_P, IND, and PHI) are necessary for preserving genetic diversity of Asian native goats.
Table 2 lists the average molecular coancestries within and among the 10 subpopulations. Molecular coancestries within each subpopulation were higher than those between the other subpopulations. Specifically, molecular coancestries within CAM_M and PHI were extremely high, indicating that these two subpopulations were affected by the larger amount of genetic drift. MGL and IND have relatively low coancestries between the other subpopulations.

Table 2. Average molecular coancestries within and among the 10 subpopulations.

\begin{tabular}{|c|c|c|c|c|c|c|c|c|c|c|}
\hline & MGL & LAO & MYA & CAM_M & CAM_P & VIE & BHU & $\mathbf{B A N}$ & IND & PHI \\
\hline MGL & 0.650 & & & & & & & & & \\
\hline LAO & 0.595 & 0.669 & & & & & & & & \\
\hline MYA & 0.597 & 0.619 & 0.628 & & & & & & & \\
\hline CAM_M & 0.559 & 0.646 & 0.624 & 0.754 & & & & & & \\
\hline CAM_P & 0.586 & 0.619 & 0.612 & 0.633 & 0.650 & & & & & \\
\hline VIE & 0.594 & 0.658 & 0.612 & 0.642 & 0.604 & 0.686 & & & & \\
\hline $\mathrm{BHU}$ & 0.615 & 0.612 & 0.611 & 0.602 & 0.604 & 0.603 & 0.641 & & & \\
\hline IND & 0.613 & 0.572 & 0.587 & 0.540 & 0.588 & 0.567 & 0.598 & 0.600 & 0.640 & \\
\hline PHI & 0.587 & 0.602 & 0.598 & 0.619 & 0.595 & 0.600 & 0.609 & 0.618 & 0.577 & 0.706 \\
\hline
\end{tabular}

Optimal genetic contributions of each subpopulation obtained by the core set method are presented in Table 3 . The maximum possible genetic diversity was estimated to be 0.398 . The subpopulations with positive contributions were the five subpopulations that were found to be genetically important in the previous analysis (MGL, MYA, CAM_P, IND, and PHI), as well as CAM_M and VIE.

Table 3. Genetic contributions of each subpopulation (ci) obtained using the core set method.

\begin{tabular}{ll}
\hline Subpopulation & $c_{i}$ \\
\hline MGL & 0.104 \\
LAO & 0.000 \\
MYA & 0.089 \\
CAM_M & 0.132 \\
CAM_P & 0.055 \\
VIE & 0.109 \\
BHU & 0.000 \\
BAN & 0.000 \\
IND & 0.410 \\
PHI & 0.101 \\
\hline
\end{tabular}

\section{Discussion}

Recalling that expected heterozygosity on the biallelic loci reduces at the rate of $1-1 / 2 N_{e}[10]$, where $N_{e}$ is the effective population size, and that standard effective population sizes of livestock breeds are around 100 [11], even a $0.5 \%$ change of heterozygosity resulting from removal of the subpopulation is not negligible. Among the five subpopulations that were found to be genetically important in the first analysis (MGL, MYA, CAM_P, IND, and PHI), the effect of MGL and IND were relatively large, and reductions were observed in both components of $G D_{\mathrm{WS}}$ and $G D_{\mathrm{BS}}$. These two subpopulations are geographically separated from the other subpopulations of Southeast Asia and are the oldest subpopulations of domestic goats introduced by nomadic tribes on the Silk Road [12].
Removal of CAM_M substantially reduced $G D_{\mathrm{BS}}$; however, it greatly increased $G D_{\mathrm{Ws}}$ and inflated $G D_{\mathrm{T}}$ as a consequence (i.e., frequencies of two alleles in each locus became equalized). This is because CAM_M has relatively large genetic distance from the other subpopulations, but also has quite small within-variability (cf. [3]). Steep mountain paths might limit the introgression of new genetic variants. A large reduction of $G D_{\mathrm{BS}}$ was also observed when PHI was removed. Geographical effect would be responsible for this reduction, i.e., PHI is an isolated island subpopulation.

In the core set analysis, the contribution of IND was fairly large, indicating that this subpopulation is predominantly important. Molecular coancestry of this subpopulation was the second lowest, and coancestries between the other subpopulations were relatively low (Table 2). India has rich goat resources; consumption of beef and pork is prohibited by Hinduism and Muslimism so goats are an important protein source [12]. The neighbor-joining tree constructed by Lin et al. [3] formed two clusters (cluster A MGL, BHU, BAN, and IND; cluster B LAO and VIE), and the remaining four subpopulations were independently distributed. MGL and IND were selected as non-zero contributors from cluster A, and VIE was selected from cluster B. This might be why BHU, BAN, and LAO could not contribute to the hypothetical gamete pool (i.e., why these subpopulations were not recognized as genetically important subpopulations).

\section{Conclusion}

Based on the analysis in this study, we concluded that the subpopulations MGL, MYA, CAM_P, IND, and PHI are primarily important for preserving the genetic diversity of Asian native goats, and that if the preservation resources remain, CAM_M and VIE are worth preserving. Compared to previous studies that uncovered origin, phylogeny, and genetic 
diversity of many livestock species in Asia using various molecular genetic techniques, this rare study provides a specific and systematic preservation scheme. In addition to neutral molecular variation, adaptive traits possessed by each subpopulation should also be taken into account in future study, as suggested by Wellmann et al. [13], and deriving the economic values of each subpopulation and designing breeding schemes is essential [14].

\section{Acknowledgements}

This work was partially supported by JSPS KAKENHI Grant Numbers 23380165 and 17H04643.

\section{References}

[1] Taberlet, P., Valentini, A., Rezaei, H. R., Naderi, S., Pompanon, F., Negrini, R., Ajmone-Marsan, P., 2008. Are cattle, sheep, and goats endangered species? Mol. Ecol. 17, 275-284.

[2] Lin, B. Z., Odahara, S., Ishida, M., Kato, T., Sasazaki, S., Nozawa, K., Mannen, H., 2012. Molecular phylogeography and genetic diversity of East Asian goats. Anim. Genet. 44, $79-85$.

[3] Lin, B. Z., Kato, T., Kaneda, M., Matsumoto, H., Sasazaki, S., Mannen, H., 2013. Genetic diversity and structure in Asian native goat analyzed by newly developed SNP markers. Anim. Sci. J. 84, 579-584.

[4] Waki, A., Sasazaki, S., Kobayashi, E., Mannen, H., 2015. Paternal phylogeography and genetic diversity of East Asian goats. Anim. Genet. 46, 337-339.
[5] Caballero, A., Toro, M., 2002. Analysis of genetic diversity for the management of conserved subdivided populations. Conserv. Genet. 3, 289-299.

[6] Nei, M., 1973. Analysis of gene diversity in subdivided populations. Proc. Natl. Acad. Sci. U. S. A. 70, 3321-3323.

[7] Nei, M., 1987. Molecular Evolutionary Genetics. Columbia University Press, New York.

[8] Eding, H., Crooijmans, R. P. M. A., Groenen, M. A. M., Meuwissen, T. H. E., 2002. Assessing the contribution of breeds to genetic diversity in conservation schemes. Gen. Sel. Evol. 34, 613-633.

[9] Mucha, S., Komen, H., 2016. Rates of inbreeding and genetic adaptation for populations managed as herds in zoos with a rotational mating system or with optimized contribution of parents. J. Anim. Breed. Genet. 133, 323-332.

[10] Gale, J. S., 1990. Theoretical Population Genetics. Unwin Hyman, London.

[11] Pirchner, F., 1983. Population Genetics in Animal Breeding. 2nd ed. Plenum Press, New York.

[12] Society for Researches on Native Livestock, Japan, 2009. Native Livestock in Asia. Nagoya University Press, Nagoya, Japan. (in Japanese).

[13] Wellmann, R., Bennewitz, J., Meuwissen, T. H. E., 2014. A unified approach to characterize and conserve adaptive and neutral genetic diversity in subdivided populations. Genet. Res. 96, e16.

[14] Biermann, A. D. M., Rommelfanger, E., Anthe, J., Frevert, H., König, S., 2016. Economic values and evaluation of breeding schemes for non-market traits with applications to an endangered pig breed. Livest. Sci. 183, 63-71. 